\title{
A QUALIDADE DOS SERVIÇOS NA HOTELARIA: UM ESTUDO COM BASE NAS ON-LINE TRAVEL REVIEWS (OTRS)
}

THE QUALITY OF HOTEL SERVICES: A STUDY BASED ON ONLINE TRAVEL REVIEWS (OTRS)

LA CALIDAD DE LOS SERVICIOS EN LA HOTELERÍA: UN ESTUDIO CON BASE EN LAS ONLINE TRAVEL REVIEWS (OTRS)

Pablo Flôres Limberger Universidade do Vale do Itajaí Docente e Pesquisador do Programa de Pós-Graduação em Turismo e Hotelaria da Universidade do Vale do Itajaí Doutor em Turismo e Hotelaria pela Universidade do Vale do Itajaí Balneário Camboriú, Santa Catarina, Brasil pablofl@univali.br

Jéssica Vieira de Souza Meira Universidade do Vale do Itajaí Bolsista do Projeto Pró-Integração, financiado pela Coordenação de Aperfeiçoamento de Pessoal de Nível Superior (CAPES) Doutoranda em Turismo e Hotelaria na Universidade do Vale do Itajaí Balneário Camboriú, Santa Catarina, Brasil jessica.meira@edu.univali.br

\section{Edar da Silva Añaña}

Universidade Federal de Pelotas Docente da Universidade Federal de Pelotas Doutor em Administração pela Universidade Federal do Rio Grande do Sul 
Pelotas, Rio Grande do Sul, Brasil

edaranana@gmail.com

\section{Ana Paula Lisboa Sohn}

Universidade do Vale do Itajaí

Docente e Pesquisadora do Programa de Pós-Graduação em Turismo e Hotelaria da Universidade do Vale do Itajaí

Doutora em Engenharia de Produção pela Universidade Federal de Santa Catarina Balneário Camboriú, Santa Catarina, Brasil

anasohn@univali.br

Data de Submissão: 20/05/2016

Data de Aprovação: 05/07/2016

RESUMO: A análise da qualidade dos serviços vem sendo objeto de estudo de diversas investigações no âmbito da hotelaria. A presente pesquisa tem como objetivo analisar a qualidade dos serviços prestados pelos hotéis de Florianópolis (SC) por meio dos critérios de avaliação da empresa Booking.com. O estudo caracteriza-se por ser descritivo, com abordagem quantitativa, realizado por meio da coleta de On-line Travel Reviews (OTRs) postadas no site www.booking. com relacionadas a 37.875 avaliações de turistas de 103 hotéis localizados em Florianópolis (SC). Os critérios avaliados foram funcionários, conforto, limpeza, custo-benefício, localização, comodidades e Wi-Fi gratuito. Utilizou-se de alguns testes estatísticos para atingir o objetivo proposto, tais como análise descritiva, análise discriminante, análise de regressão e análise de cluster. Os resultados alcançados demonstram que os critérios relacionados ao conforto e às comodidades influenciam na avaliação dos demais critérios, bem como na avaliação da satisfação geral realizada pelos turistas.

PALAVRAS-CHAVE: Qualidade dos Serviços, Hotelaria, On-line Travel Reviews, Booking.com.

ABSTRACT: The quality of services offered by hotels has been the object of several studies in the area of hotel management. The main purpose of this research is to analyse the quality of services provided by hotels in Florianópolis (SC), through the evaluation criteria of the company Booking. com. The study uses a descriptive-quantitative approach, based on Online Travel Reviews (OTRs) available on the website www.booking.com. A total of 103 hotels located in Florianopolis (SC) were evaluated by 37,875 tourists. The criteria evaluated were staff performance, comfort, cleanliness, value for money, location, amenities and free Wi-Fi. We used some statistical tests to achieve the proposed objective, such as descriptive analysis, discriminant analysis, multiple regression analysis, and cluster analysis. The results show that the criteria comfort and amenities influence the evaluation of the other criteria, as well as the tourists' overall satisfaction.

KEYWORDS: Service Quality, Hotel, Online Travel Reviews, Booking.com. 
RESUMEN: El análisis de la calidad de los servicios viene siendo objeto de estudio de diversas investigaciones en el ámbito de la hotelería. Esta investigación tiene como objetivo analizar la calidad de los servicios prestados por los hoteles de Florianópolis (SC), utilizando los criterios de evaluación de la empresa Booking.com. El estudio se caracteriza como descriptivo, con enfoque cuantitativo, realizado a través de la recopilación de Online Travel Reviews (OTRs) publicada en el sitio web www.booking.com, relacionada a 37.875 opiniones de los turistas de 103 hoteles ubicados en Florianópolis (SC). Los criterios evaluados fueron el personal, la comodidad, la limpieza, la relación calidad-precio, ubicación, instalaciones y conexión Wi-Fi. Se utilizaron pruebas estadísticas para lograr el objetivo propuesto, tales como el análisis descriptivo, análisis discriminante, análisis de regresión y análisis de conglomerados. Los resultados muestran que los criterios relacionados con el confort y las instalaciones influyen en la evaluación de los otros criterios, así como en la evaluación de la satisfacción general realizada por los turistas.

PALABRAS CLAVE: Calidad de los Servicios; Hotel; Online Travel Reviews; Booking.com.

\section{INTRODUÇÃO}

crescente dependência da internet como fonte de informação para
fazer escolhas sobre produtos turísticos levanta a necessidade de
mais pesquisas sobre os novos modelos de negócio que surgem baseados na inovação tecnológica, como é o caso da empresa Booking.com. Neste sentido, o presente estudo tem como objetivo analisar a qualidade dos serviços prestados pelos hotéis de Florianópolis (SC), através dos critérios de avaliação da empresa Booking.com.

O Booking.com faz parte do grupo Priceline e é líder em reservas on-line de meios de hospedagem. Segundo o site da empresa, todos os dias mais de um milhão de diárias são reservadas através do Booking.com. O site e os aplicativos do Booking.com atraem visitantes de todo o mundo, interessados tanto no mercado de turismo de lazer quanto de negócios. A empresa foi fundada em 1996 em Amsterdã, na Holanda, estando disponível em mais de 40 idiomas, sendo parceira de mais de 900.000 meios de hospedagem, em 223 países (Booking.com 2016). O Booking.com, além de ser um site de reservas, é uma Online Travel Review (OTR), ou seja, uma mídia social cujos usuários postam comentários sobre viagens (Xiang \& Gretzel, 2010).

A cidade de Florianópolis, capital do estado de Santa Catarina, possui um pouco mais de 469 mil habitantes, distribuídos em uma área de $675 \mathrm{Km}^{2}$. Banhada 
pelo Oceano Atlântico, o destino conta com mais de 100 praias, de belezas naturais singulares. O setor de serviços - liderado pela atividade turística - é o principal gerador de divisas para a economia local (Instituto Brasileiro de Geografia e Estatística, 2016; Portal da Ilha, 2016). Florianópolis foi considerada, no ano de 2015, pela Organização das Nações Unidas $\mathrm{ONU}$, a capital brasileira com a mais alta qualidade de vida e a quarta melhor cidade do país para se viver, com o terceiro melhor índice de desenvolvimento humano (Prefeitura Municipal de Florianópolis, 2016; Portal da Ilha, 2016).

Observa-se que publicações em importantes journals têm destacado a importância de se estudar novos modelos de negócio, como o Booking.com e sua relação com a satisfação do consumidor. O estudo de Ye, Law e Gu (2009) investigou as variáveis relacionadas aos comentários dos consumidores do Booking.com. Os resultados sugerem que os consumidores tendem a avaliar um hotel com base em comentários de outros hóspedes, respaldando a importância de estudos sobre qualidade de serviços por meio da avaliação dos consumidores em empresas como a Booking.com. Outros estudos que realçam a importância desta temática estão apresentados na pesquisa teórica que segue a introdução.

Para o alcance do objetivo proposto, o artigo está organizado em seções. $\mathrm{Na}$ introdução tem-se a apresentação do contexto e do objetivo da pesquisa. Esta seção é seguida pela pesquisa teórica sobre duas importantes temáticas que subsidiaram a análise dos resultados: qualidade dos serviços na hotelaria e qualidade dos serviços hoteleiros nas On-line Travel Reviews (OTRs). Em seguida tem-se a descrição da metodologia da pesquisa e a análise e a discussão dos resultados. Por fim são apresentadas as considerações finais, destacando o alcance do objetivo proposto e as contribuições deste estudo para outras investigações no campo do turismo.

\section{QUALIDADE DOS SERVIÇOS NA HOTELARIA}

A temática voltada à qualidade dos serviços hoteleiros vem sendo objeto de estudo de diversas pesquisas atualmente. Muitos autores à relacionam com a satisfação dos hóspedes (Blesic, Ivkov-Dzigurski, Stankov, Stamenkovic, 
\& Bradic, 2011; Curakovic, Sikora, Garaca, Curcic, \& Vukosav, 2013; Markovic \& Jankovic, 2013), outros com a lealdade (Kuo, Chang, Cheng, \& Lai, 2013; Wilkins, Merrilees, \& Herington, 2010), existem os que defendem a utilização da qualidade como ferramenta gerencial (Alonso-Almeida, Rodríguez-Antón, \& Rubio-Andrada 2012), ou como vantagem competitiva (Silva \& Teixeira 2007; Zhong, Chen \& Xie, 2010). O comportamento do hóspede também é relacionado constantemente com a qualidade dos serviços hoteleiros (Kuo, Chang, Chen, \& Hsu, 2012; Zhen \& Zhu, 2010), bem como as suas percepções (Grobelna \& Marciszewska, 2013; Johann \& Anastassova 2014; Petry, Pickler, \& Tomelin, 2016; Stupariu \& Josan, 2014).

Uma ferramenta comumente utilizada para avaliar a qualidade dos serviços prestados por hotéis é o SERVQUAL, criada por Parasuraman, Zeithaml e Berry em 1988, que realiza a mensuração do grau de satisfação com relação ao grau de expectativa. Segundo Berry e Parasuraman (1995), esta ferramenta serve para avaliar os pontos forte e fracos por meio de cinco dimensões: tangibilidade (aspectos físicos), confiabilidade (habilidade de prestar o serviço prometido com exatidão), capacidade de resposta (disposição para auxiliar os clientes com responsabilidade), segurança (conhecimento, cortesia e capacidade de transmitir confidencialidade) e empatia (disposição em demonstrar interesse e atenção). Os autores acreditam que as expectativas dos clientes desempenham um papel fundamental na avaliação dos serviços prestados pela empresa, os quais influenciam na satisfação dos mesmos.

A escala SERVQUAL vem sofrendo algumas adaptações ao longo dos anos, direcionadas a situações específicas, a fim de ser utilizada da melhor forma possível pelos mais diversos setores da economia. Pode-se citar como exemplo a escala SERVFERF (Cronin \& Taylor, 1992), a qual avalia a qualidade apenas através das percepções dos consumidores com relação aos serviços prestados. O LODGSERV (Knutson, Stevens, Wullaert, Patton, \& Yokoyama, 1990), o HOTELSERV (Mei, Dean, \& White, 1999) e o HOTELQUAL (Falces, Sierra, Becerra, \& Briñol, 1999) são outros três modelos de adaptações do SERVQUAL, os quais foram criados especialmente para a indústria hoteleira. Embora o SERVQUAL ainda seja a escala mais utilizada, estes modelos foram criados com o objetivo de fazer com que as particularidades dos hotéis 
sejam atendidas de maneira otimizada, tendo em vista a competitividade do mercado (Souza, Meira, \& Maske, 2012).

Existem alguns estudos acadêmicos que utilizaram a escala SERVQUAL e suas adaptações. A investigação de Law e Yip (2010) examinou o nível de satisfação dos turistas em um hotel da China, através da aplicação de questionários com os seus hóspedes, e constatou que os mesmos se encontram satisfeitos com os serviços oferecidos pelo hotel, destacando o valor medicinal como o seu principal atrativo. Os autores Blesic et al. (2011) analisaram a medição da qualidade dos serviços no setor hoteleiro da Sérvia. Por meio da aplicação de questionários com hóspedes e da utilização de uma escala baseada no SERVQUAL, constataram que os hóspedes não estão satisfeitos com os serviços dos hotéis. Suas expectativas foram maiores do que suas percepções em todos os fatores, exceto no fator empatia, cujo valor positivo da lacuna é resultado de baixas expectativas.

O estudo de Lima-Filho, Marchiotti e Quevedo-Silva (2012) mensurou a satisfação dos hóspedes dos hotéis de Campo Grande/MS, também através da aplicação do SERVQUAL. Os autores concluíram que a maioria dos hóspedes estão satisfeitos com a maior parte das dimensões avaliadas. Aspectos como instalações, atendimento, empatia e confiabilidade foram bem avaliados. Entretanto, aspectos como segurança e alimentação tiveram avaliações negativas. A pesquisa de Grobelna e Marciszewska (2013) utilizou uma adaptação do SERVQUAL para analisar a percepção dos hóspedes e dos gestores quanto à qualidade dos serviços prestados pelos hotéis da Polônia. Os autores constataram, após aplicação de questionários, que os aspectos voltados à confiabilidade e à empatia receberam avaliações negativas tanto dos hóspedes quanto dos gestores hoteleiros.

A investigação de Kuo et al. (2013) buscou identificar a influência da qualidade do serviço na lealdade do cliente na indústria hoteleira de Taiwan através da escala SERVQUAL. Os autores identificaram que a satisfação do cliente atua como variável mediadora entre a qualidade do serviço e a lealdade. A pesquisa de Markovic e Jankovic (2013) examinou a relação entre qualidade do serviço e satisfação do cliente dos hotéis da Croácia, através da escala SERVQUAL adaptada. Os autores concluíram que as principais dimensões da qualidade 
percebida são: confiabilidade, empatia, competência, acessibilidade e aspectos tangíveis. O estudo de Ban e Mester (2014) também utilizou a escala SERVQUAL adaptada com o intuito de avaliar a qualidade global dos hotéis da Romênia. Os autores concluíram que a maioria dos entrevistados avalia a satisfação global de forma positiva.

Alguns estudos abordaram a temática voltada à qualidade dos serviços através da utilização de outros modelos, métodos e escalas, defendendo a ideia de que a qualidade dos serviços auxilia na competitividade dos hotéis (Marinescu \& Ispas, 2012; Silva \& Teixeira, 2007). A pesquisa de Wilkins, Merrilees e Herington (2010) aplicou questionários com hóspedes de hotéis localizados na Austrália, através da utilização do método estatístico de análise fatorial. Os autores concluíram que a qualidade dos serviços, mediada pela satisfação do hóspede, é considerada a maior determinante da lealdade dos mesmos. A investigação de Kuo et al. (2012), realizada nos hotéis da China, também utilizou a análise fatorial para comprovar suas hipóteses de pesquisa, chegando à mesma conclusão que o estudo de Wilkins, Merrilees e Herington (2010), ou seja, que a satisfação serve como mediadora entre a qualidade dos serviços e a lealdade.

O estudo de Curakovic et al. (2013) buscou investigar a qualidade dos serviços oferecidos pelos hotéis da Sérvia e concluíu que, embora tenha apresentado um alto nível de satisfação dos hóspedes, a alimentação obteve níveis mais baixos do que o esperado. Por outro lado, os autores Johann e Anastassova (2014), através da aplicação de questionários com hóspedes de hotéis da Bulgária, identificaram os serviços de alimentação como os melhores avaliados, juntamente com a padronização, a localização, as praias, o entretenimento e o serviço ao cliente. A pesquisa de Stupariu e Josan (2014) também buscou analisar a qualidade dos serviços prestados por hotéis, neste caso, localizados na Romênia. Através da aplicação de questionários com os hóspedes, os autores identificaram lacunas na padronização, no marketing, no treinamento da equipe, na informação ao turista, na limpeza e na reciclagem de lixo.

A pesquisa de Chen e Tseng (2012) trabalhou com a formulação de hipóteses para analisar a importância do treinamento nos hotéis de Taiwan. Através da aplicação de questionários com funcionários, os autores identificaram a 
qualidade dos serviços como um fator fundamental para o melhor desempenho dos hotéis pesquisados. O estudo de Li (2014) também avaliou a qualidade dos serviços de uma forma um pouco diferente do habitual. Através da aplicação de questionários com os hóspedes de hotéis localizados na China e utilização das técnicas estatísticas de análise fatorial e modelagem de equação estrutural, o autor analisou a relação entre o comportamento de outros clientes e a avaliação da qualidade do serviço percebida pelos hóspedes. Como conclusão, constatou que o comportamento dos outros clientes exerce influência direta sobre a qualidade dos serviços percebida pelos hóspedes.

\section{QUALIDADE DOS SERVIÇOS HOTELEIROS NAS ON-LINE}

TRAVEL REVIEWS

Investigações relacionadas à qualidade dos serviços hoteleiros através da utilização das informações disponibilizadas pelas On-line Travel Reviews (OTRs) têm interessado os pesquisadores do turismo, entre as quais se podem destacar: Jeong e Jeon (2008); Lee e Hu (2008); Barcala, Díaz e Rodriguez (2009); Black e Kelly (2009); Zheng, Youn e Kincaid (2009); O'Connor (2010); Stringam e Gerdes Jr. (2010); Stringam, Gerdes Jr. e Vanleeuwen (2010); Zehrer, Crotts e Magnini (2011); Ong (2012); Browning, So e Sparks (2013); Chaves, Gomes e Pedron (2013); Gidumal, González e Valcarcel (2013); González, Gidumal e Valcarcel (2013); Levy, Duan e Boo (2013); Li, Ye e Law (2013); Liu, Law, Rong, Li \& Hall (2013); Noone e McGuire (2013); Jiang, Gretzel e Law (2014); Limberger, Anjos, Meira e Anjos (2014); Linnes, Kowalski, Lema, Lam e Agrusa (2014); Gémar e Quintero (2015); Petry, Pickler e Tomelin (2016).

Os turistas que utilizam as OTRs costumam confiar nas avaliações de outros usuários, conforme os resultados obtidos pela pesquisa de Linnes et al. (2014). Os comentários que transmitem confiança normalmente são ricos em detalhes (Zehrer, Crotts, \& Magnini, 2011), sendo que os comentários que descrevem uma recuperação efetiva 'do um erro' são considerados os mais úteis (Black \& Kelley, 2009). Os usuários das OTRs também costumam prestar atenção no perfil do comentarista e na data do comentário (Ong, 2012). A quantidade de comentários nas OTRs tem uma relação positiva com as avaliações do hotel, segundo a pesquisa 
de González, Gidumal e Valcarel (2013), por isso a tendência é que quanto maior o número de comentários, mais positiva deverá ser a avalição.

Na pesquisa de Browning, So e Sparks (2013) foi identificado que os consumidores são mais propensos a fazer comentários positivos sobre um conjunto de serviços, do que somente sobre os serviços desempenhados pelos funcionários. Entretanto, é importante ressaltar que, apesar das avaliações serem voltadas ao serviço hoteleiro, a satisfação do hóspede é impactada em $11,38 \%$ pela satisfação com relação ao destino turístico, conforme pesquisa de Gidumal, González e Valcarcel (2013). A satisfação geral relacionada ao serviço hoteleiro é normalmente consistente com as subcategorias (serviço, condições do hotel, limpeza e conforto), conforme Stringam, Gerdes Jr. e Vanleeuwen (2010). A pesquisa de Liu et al. (2013) identificou que 97\% da variância na satisfação pode ser explicada pelos critérios: quarto, valor, limpeza, check-in e check-out.

A maioria dos artigos que trabalha com a avaliação da qualidade dos serviços prestados pelos hotéis associa a satisfação dos turistas com os critérios de avaliação: quarto, serviços (funcionários), conforto, limpeza, custo-benefício, localização e comodidades. O critério quarto compôs a satisfação na hotelaria nas seguintes investigações: Jeong e Jeon (2008), O'Connor (2010), Ong (2012), Chaves, Gomes e Pedron (2013), Levy, Duan e Boo (2013), Liu et al. (2013), Jiang, Gretzel e Law (2014), Limberger et al. (2014) e Petry, Pickler e Tomelin (2016). A pesquisa de Jeong e Jeon (2008) identificou o quarto como sendo um dos principais critérios de avaliação na hotelaria.

Os seguintes estudos consideraram o critério serviço como relevante: Jeong e Jeon (2008), Lee e Hu (2008), Barcala, Díaz e Rodriguez (2009), Zheng, Youn e Kincaid (2009), O'Connor (2010), Stringam, Gerdes Jr. e Vanleeuwen (2010), Ong (2012), Chaves, Gomes e Pedron (2013), Levy, Duan e Boo (2013), Liu et al. (2013), Li, Ye e Law (2013), Jiang, Gretzel e Law (2014) e Limberger et al. (2014). Para os pesquisadores Jeong e Jeon (2008), a prestação do serviço explica 97\% da variância na satisfação dos hóspedes. Segundo os resultados encontrados por Ong (2012), 66\% dos leitores dos comentários das OTRs consideram relevantes as opiniões relacionadas aos serviços prestados pelos funcionários do hotel. 
O conforto foi considerado relevante para a satisfação na hotelaria nas seguintes pesquisas: O'Connor (2010), Stringam e Gerdes Jr. (2010), Stringam, Gerdes Jr. e Vanleeuwen (2010), Chaves, Gomes e Pedron (2013), Liu et al. (2013), Jiang, Gretzel e Law (2014) e Limberger et al. (2014). Para Stringam, Gerdes Jr. e Vanleeuwen (2010), o critério conforto, juntamente com os serviços, são considerados os principais influenciadores na satisfação geral. 0 conforto é um critério que normalmente recebe avaliações positivas, conforme O'Connor (2010). Contrariando esta linha de pensamento, Stringam e Gerdes Jr. (2010) identificaram na sua pesquisa, comentários negativos relacionados a este critério. O estudo de Chaves, Gomes e Pedron (2013), corroborando com Stringam e Gerdes Jr. (2010), identificou o critério conforto como o mais comumente relacionado aos comentários negativos.

A limpeza é considerada um critério de avaliação da satisfação no setor hoteleiro para os seguintes pesquisadores: Jeong e Jeon (2008), O'Connor (2010), Stringam, Gerdes Jr. e Vanleeuwen (2010), Liu et al. (2013), Jiang, Gretzel e Law (2014) e Limberger et al. (2014). O estudo de Jeong e Jeon (2008) considerou a limpeza como um dos principais critérios de avaliação. É importante ressaltar que, quando a limpeza recebe uma avaliação baixa, é quase impossível o hotel receber uma avaliação geral alta (Stringam, Gerdes Jr., \& Vanleeuwen, 2010).

Os investigadores que abordaram o critério custo-benefício foram os seguintes: Jeong e Jeon (2008), Lee e Hu (2008), Barcala, Díaz e Rodriguez (2009), Ong (2012), Liu et al. (2013), Noone e McGuire (2013), Jiang, Gretzel e Law (2014). O custo-benefício é considerado um dos principais critérios de avaliação e representa, junto com o serviço, o quarto e a limpeza, 97\% da variância, sendo considerado o custo-benefício o principal critério para a satisfação (Jeong \& Jeon, 2008). Para 69\% dos leitores, o custo-benefício é considerado um critério importante (Ong, 2012). Contrapondo, a pesquisa de Noone e McGuire (2013) não identificou uma relação significativa entre o preço e a avaliação da satisfação na fase anterior à compra. Entretanto, na investigação de Lee e Hu (2008), a cobrança acima do combinado foi uma das principais categorias de reclamações. 
Pesquisadores como Jeong e Jeon (2008), O'Connor (2010), Ong (2012), Chaves, Gomes e Pedron (2013), Liu et al. (2013), Jiang, Gretzel e Law (2014), Limberger et al. (2014) e Petry, Pickler e Tomelin (2016) consideraram a localização como um critério relevante para a análise da satisfação no setor hoteleiro. Foi identificado por O'Connor (2010) que a variável localização para a experiência do serviço é mais mencionada dentre os comentários positivos. Nas pesquisas de Jeong e Jeon (2008) e Limberger et al. (2014) a localização não apresentou relação forte com a satisfação geral.

As comodidades foram consideradas critérios influenciadores na avaliação da satisfação geral nas pesquisas desenvolvidas por Barcala, Díaz e Rodriguez (2009), Stringam, Gerdes Jr. e Valeeuwen (2010), Levy, Duan e Boo (2013), Li, Ye e Law (2013) e Jiang, Gretzel e Law (2014). No estudo de Stringam, Gerdes Jr e Valeeuwen (2010) foi identificada uma relação positiva entre as comodidades e a satisfação geral. Corroborando com este resultado, a pesquisa de Barcala, Díaz e Rodriguez (2009) identificou que quanto mais antigas forem as comodidades, mais esperada é a redução da satisfação dos hóspedes.

\section{METODOLOGIA}

A presente investigação, de caráter descritivo e abordagem quantitativa, busca analisar a qualidade dos serviços prestados pelos hotéis de Florianópolis (SC), através dos critérios de avaliação dos turistas identificados no site www. booking.com. A escolha de Florianópolis, capital do estado de Santa Catarina, ocorreu em virtude da importância que a atividade turística exerce na economia local. Florianópolis faz parte dos 65 destinos indutores do turismo no Brasil, sendo considerado pelo TripAdvisor (2016) como o sétimo melhor destino turístico do Brasil. O Booking.com foi escolhido pelo fato de ser considerado o líder mundial em reservas de hospedagem on-line (Borges, Pereira, Matos, \& Borchardt, 2015).

A coleta das informações junto ao Booking.com ocorreu no mês de abril de 2016 e foi realizada manualmente. Foram encontradas 37.875 avaliações de turistas relativas a 103 hotéis, entre 01 e 05 estrelas, localizados em Florianópolis 
(SC). As médias das avaliações a respeito das experiências dos turistas com relação aos hotéis estão disponibilizadas em uma escala de notas de 0 a 10 . Os critérios avaliados pelos turistas são relacionados aos funcionários (serviços), conforto, limpeza, custo-benefício, localização, comodidades e Wi-Fi gratuito. O sítio também fornece uma nota de satisfação geral, classificada entre 0 e 10. Para tabulação e análise das informações coletadas junto ao Booking.com, utilizou-se o software SPSS versão 22.

A análise dos dados foi realizada, primeiramente, através de testes estatísticos descritivos, com o objetivo de verificar a distribuição dos hotéis conforme a classificação por estrelas, bem como para identificar os critérios mais bem avaliados pelos turistas e, com isso, apresentar uma caracterização inicial da situação da hotelaria em Florianópolis (SC). Em um segundo momento, foi realizada a análise discriminante, para verificar se a classificação por estrelas tem relação com a qualidade dos serviços. Esta técnica multivariada permite a redução da dimensionalidade dos dados, distribuindo-os em grupos distintos (Hair Jr., Anderson, Tatham, \& Black, 2009), neste caso, a classificação por estrelas.

A análise de regressão múltipla foi utilizada para avaliar a relevância dos diversos critérios para a avaliação geral. Como variável dependente foi utilizada a nota relacionada à satisfação geral e como variáveis independentes foram utilizados os critérios de avaliação (funcionários, conforto, limpeza, custobenefício, localização, comodidades e Wi-Fi gratuito). Por último foi realizada uma análise de cluster, com vistas a agrupar casos com perfis similares protótipos -, buscando maximizar a homogeneidade interna dos objetos e a heterogeneidade externa entre os grupos (Hair Jr et al., 2009; Martins \& Theóphilo, 2009).

\section{ANÁLISE E DISCUSSÃO DOS RESULTADOS}

Os 103 hotéis de Florianópolis (SC) identificados no Booking.com encontramse divididos entre as categorias de 01 a 05 estrelas, conforme informações disponibilizadas na Tabela 01. Através da análise descritiva - frequência e média 

- pode-se perceber que os hotéis classificados com 03 estrelas são os mais ofertados (51,5\%), seguidos pelos hotéis de categoria 04 estrelas (33\%), ou seja, essas duas classificações hoteleiras representam $84,5 \%$ (87 hotéis) da oferta total dos meios de hospedagem de Florianópolis (SC).

Tabela 01: Número de hotéis conforme a classificação por estrelas.

$\begin{array}{ccc}\text { Classificação } & \text { Frequência (N) } & \text { Percentual (\%) } \\ \text { 01 Estrela } & 01 & 1,0 \\ \text { 02 Estrelas } & 10 & 9,7 \\ \text { 03 Estrelas } & 53 & 51,5 \\ \text { 04 Estrelas } & 34 & 33,0 \\ \text { 05 Estrelas } & 05 & 4,9 \\ \text { Total Geral } & \mathbf{1 0 3} & \mathbf{1 0 0 , 0}\end{array}$

Fonte: Dados da Pesquisa (Booking.com 2016).

Para analisar os critérios de avaliação dos turistas, também foi utilizada a estatística descritiva através do cálculo das médias. Os resultados apresentam um certo equilíbrio entre os valores das avaliações, variando entre a menor média que está relacionada ao serviço de Wi-Fi gratuito $(6,53)$ e a maior média, relacionada à localização (8,37). Os estudos de O'Connor (2010), Johann e Anastassova (2014) e Petry, Pickler e Tomelin (2016) também identificaram comentários positivos relacionados ao critério localização. Outro critério que obteve uma média alta está relacionado aos funcionários (8,12\%), corroborando com os estudos de Jeong e Jeon (2008), Stringam, Gerdes Jr. e Vanleeuwen (2010), Lima-Filho, Marchiotti e Quevedo-Silva (2012) e Johann e Anastassova (2014). A Tabela 02 apresenta os valores das médias dos critérios.

Tabela 02: Média dos critérios de avaliação dos turistas.

$\begin{array}{cc}\text { CRITÉRIOS } & \text { MÉDIAS (NOTAS) } \\ \text { Funcionários } & 8,12 \\ \text { Conforto } & 7,50 \\ \text { Limpeza } & 7,79 \\ \text { Custo-Benefício } & 7,48 \\ \text { Localização } & 8,37 \\ \text { Comodidades } & 7,49 \\ \text { Wi-Fi Gratuito } & 6,53\end{array}$

Fonte: Dados da Pesquisa (Booking.com 2016).

De acordo com a análise discriminante, pode-se afirmar que a classificação hoteleira, baseada na tipologia de estrelas, apresentou-se como um meio adequado para categorizar os hotéis. Diferentemente dos resultados obtidos pela pesquisa Grobelna e Marciszewska (2013), que não conseguiu identificar diferenças entre 
as classificações por estrelas, o resultado do presente trabalho sugere que aquela classificação acompanha razoavelmente os indicadores aqui avaliados.

Conforme pode ser comprovado na Figura 01, a separação das categorias através dos indicadores de qualidade aqui analisados apresenta pequenas áreas de sombreamento entre elas e os centroides de cada grupo apresentamse bem separados uns dos outros. O hotel classificado com 01 estrela foi omitido da figura, por não ter representatividade estatística. Percebe-se também que as classificações hoteleiras seguem uma ordem crescente, ou

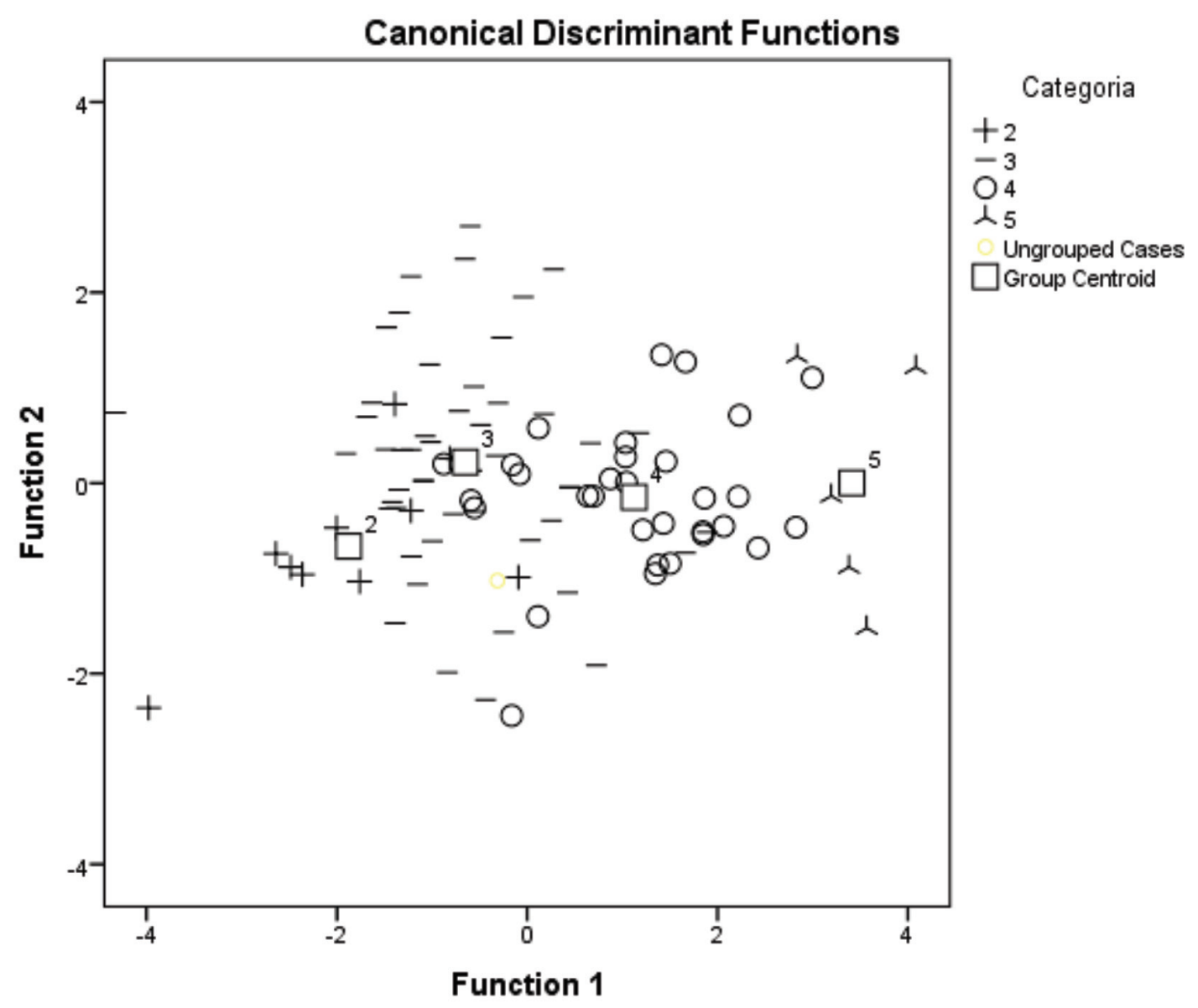

seja, os hotéis 02 estrelas possuem as avaliações mais baixas, seguidos pelos hotéis de 03 estrelas e 04 estrelas. Os hotéis classificados com 05 estrelas possuem as avaliações mais altas.

Figura 01: Análise canônica discriminante.

Fonte: Dados da Pesquisa (Booking.com 2016).

Através da análise de regressão, constatou-se que todos os critérios, com exceção do Wi-Fi gratuito, apresentaram significância estatística para a formação da satisfação geral dos turistas. Este resultado corrobora os estudos anteriores, haja visto que a oferta de Wi-Fi gratuito já não aparece nas 
pesquisas anteriores como variável formadora de satisfação. Uma hipótese que se pode levantar a partir dos resultados obtidos é que o oferecimento de Wi-Fi gratuito já não seja mais considerado um diferencial de qualidade na visão dos turistas, mas sim uma obrigatoriedade, uma espécie de padrão mínimo nas instalações hoteleiras. Os resultados obtidos através da análise de regressão múltipla, realizada pelo Método Stepwise, apontam que, após a inclusão das seis variáveis independentes, o modelo apresenta $R^{2}$ igual 0,998, o que pode ser interpretado como um sinal de multicolinearidade, uma circunstância que, conforme Hair Jr. et al. (2009, p.213), "pode distorcer os resultados ou torná-los instáveis".

A Tabela 03 apresenta os resultados obtidos através da análise de regressão múltipla, em que se percebe que os critérios relacionados à comodidade (Beta:0,220), ao conforto (Beta: 0,218) e à limpeza (Beta: 0,191) tiveram um impacto maior do que os demais critérios na avaliação geral do serviço. Em outras palavras, é possível dizer que, para cada ponto que seja agregado na comodidade do hotel, agregar-se-á 0,220 ponto na avaliação da satisfação geral; e que cada ponto extra que se agregue ao conforto, transferirá o equivalente a 0,218 ponto na avaliação da satisfação geral, e assim por diante. Nota-se também que o critério menos impactante está relacionado aos funcionários, correspondendo 0,160 ponto na satisfação geral, a cada ponto.

Tabela 03: Coeficientes do modelo de regressão

\begin{tabular}{|c|c|c|c|c|c|}
\hline \multirow{2}{*}{$\begin{array}{l}\text { Variáveis Incluídas no } \\
\text { Modelo }\end{array}$} & \multicolumn{2}{|c|}{$\begin{array}{c}\text { Coeficientes Não } \\
\text { Padronizados }\end{array}$} & \multirow{2}{*}{$\begin{array}{c}\text { Coeficientes } \\
\text { Padronizados } \\
\text { Beta }\end{array}$} & \multirow[t]{2}{*}{$t$} & \multirow[t]{2}{*}{ Significância } \\
\hline & $B$ & Erro Padrão & & & \\
\hline (Constant) & 0,04 & 0,05 & - & 0,83 & 0,41 \\
\hline Comodidades & 0,18 & 0,03 & 0,22 & 6,63 & 0,00 \\
\hline Funcionários & 0,16 & 0,01 & 0,16 & 15,38 & 0,00 \\
\hline Localização & 0,16 & 0,01 & 0,17 & 24,56 & 0,00 \\
\hline Custo-Benefício & 0,16 & 0,01 & 0,17 & 18,72 & 0,00 \\
\hline Limpeza & 0,16 & 0,01 & 0,19 & 13,34 & 0,00 \\
\hline Conforto & 0,17 & 0,02 & 0,22 & 7,05 & 0,00 \\
\hline
\end{tabular}

a. Dependent Variable: Nota Geral

Fonte: Dados da Pesquisa (Booking.com 2016). 
Os resultados obtidos na Tabela 03 corroboram com as pesquisas de Barcala, Díaz e Rodriguez (2009), Stringam, Gerdes Jr. e Valeeuwen (2010), Lima-Filho, Marchiotti e Quevedo-Silva (2012), Levy, Duan e Boo (2013), Li, Ye e Law (2013) e Jiang, Gretzel e Law (2014) quanto ao critério comodidades, uma vez que também identificaram este critério como grande influenciador da satisfação geral. O critério conforto teve destaque nas pesquisas de O'Connor (2010), Stringam e Gerdes Jr. (2010), Stringam, Gerdes Jr. e Vanleeuwen (2010), Chaves, Gomes e Pedron (2013), Liu et al. (2013), Jiang, Gretzel e Law (2014) e Limberger et al. (2014), os quais identificaram este critério como um dos maiores influenciadores na satisfação geral.

A análise de cluster foi utilizada para agrupar as avaliações dos turistas que possuem características semelhantes entre si. O dendograma - apresentado na Figura 02 - obtido com o algoritmo hierarchical cluster analysis presente no pacote SPSS sugere a existência de três clusters de respondentes, sendo estes formados por um grande grupo e dois grupos menores.

Figura 02: Dendograma da análise de cluster

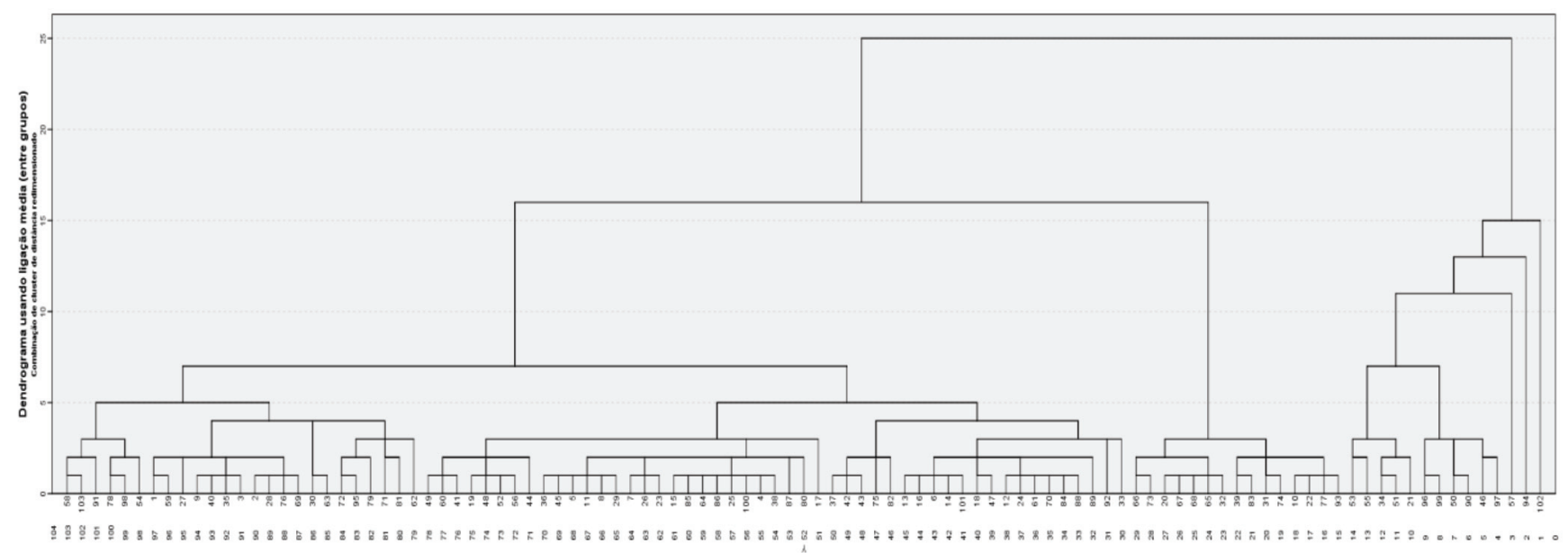

Fonte: Dados da Pesquisa (Booking.com 2016)

Após a identificação dos três clusters, estes foram agrupados pelos valores médios de cada critério (funcionários, conforto, limpeza, custo-benefício, localização, comodidades) e transformados em gráfico. O objetivo foi identificar as diferenças entre os grupos, bem como os critérios que influenciam na avaliação dos demais. A Figura 03 apresenta um gráfico com os valores médios de cada critério, em cada um dos três grupos, separadamente. 
Figura 03: Média dos critérios nos três agrupamentos

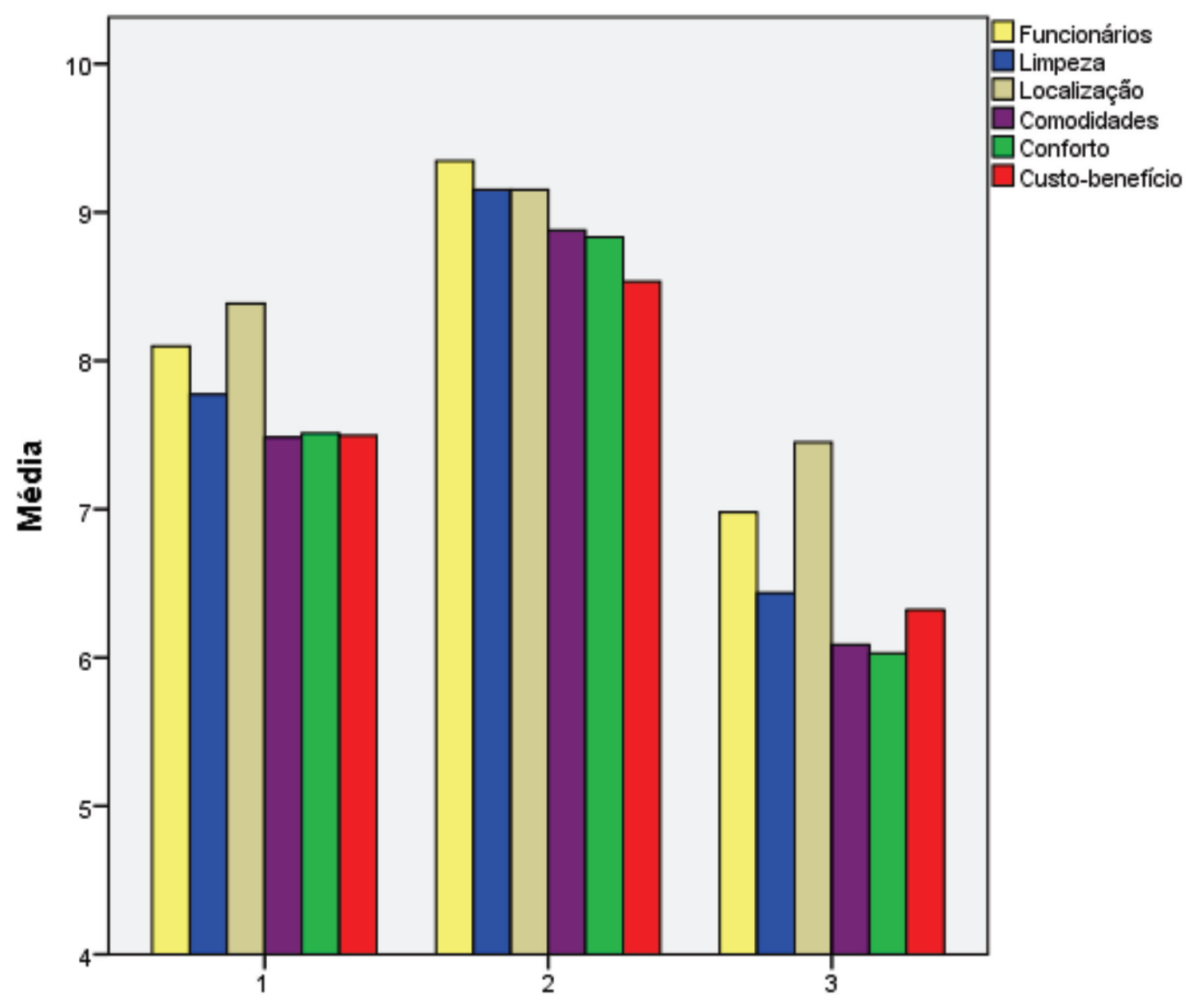

Average Linkage (Between Groups)

Fonte: Dados da Pesquisa (Booking.com 2016).

Conforme as informações apresentadas na Figura 03, nota-se que os três grupos têm como base um conjunto de critérios com resultados, em sua maioria, condizentes entre si. Desta forma, percebe-se que o grupo 01 recebeu avaliações, em sua maioria, entre as notas 7 e 8 . No grupo 02, as avaliações estão perto da nota 9 e no grupo 3 as avaliações se concentram entre as notas 6 e 7.

Inicialmente, podem-se destacar os critérios localização e funcionários, os quais tendem a receber uma avaliação superior em relação aos demais critérios. Estes resultados corroboram com as pesquisas de O'Connor (2010) e Johann e Anastassova (2014), todavia, nas investigações de Jeong e Jeon (2008) e Limberger et al. (2014), o critério localização não apresentou uma relação forte com a satisfação geral. O critério relacionado aos funcionários também alcançou avaliações positivas nas pesquisas de Jeong e Jeon (2008), Stringam, Gerdes Jr. 
e Vanleeuwen (2010), Lima-Filho, Marchiotti e Quevedo-Silva (2012), Johann e Anastassova (2014). Entretanto, os autores Stupariu e Josan (2014) encontraram lacunas em sua investigação relacionadas ao treinamento dos funcionários.

Os critérios relacionados ao conforto e à comodidade mostram-se, novamente, com significativo impacto na avaliação da satisfação geral realizada pelos turistas, uma vez que eles influenciam nas avaliações dos demais critérios, tendenciando-os a terem avaliações similares. Este fato demonstra que quando o conforto e a comodidade são mal avaliados pelos turistas, é pouco provável que o hotel tenha uma avaliação da satisfação geral positiva. Da mesma forma, quando o conforto e a comodidade apresentam avaliações positivas, é bem provável que o hotel tenha uma avaliação da satisfação geral dos turistas positiva.

Para aprofundar a análise dos resultados obtidos através da Figura 03, foi elaborado um gráfico de dispersão, agrupando os critérios comodidade e conforto no eixo vertical e os demais critérios no eixo horizontal. O resultado obtido apresenta uma relação positiva dos critérios conforto e comodidade com os demais critérios, ou seja, quanto maior for a avaliação destes dois critérios, maior será a avaliação dos demais, comprovando a discussão gerada através da análise da Figura 03. O gráfico de dispersão encontra-se na Figura 04.

Figura 04: Gráfico de dispersão dos critérios conforto e comodidade

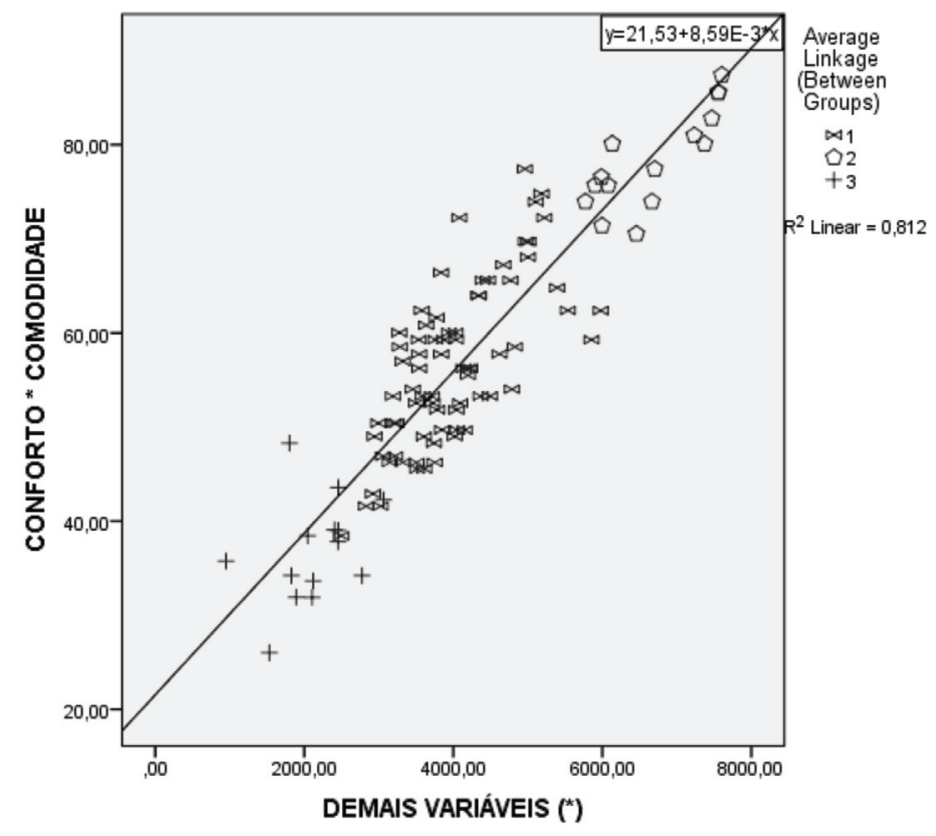

Fonte: Dados da Pesquisa (Booking.com 2016). 
Através da análise da Figura 04, percebe-se que os agrupamentos continuaram na mesma ordem de dispersão, ou seja, o grupo 02 possuindo as melhores avaliações dos turistas; o grupo 03, as piores; e o grupo 01, as avaliações medianas, demonstrando que realmente o conforto e a comodidade são os critérios que influenciam na avaliação dos demais e, consequentemente, na avaliação da satisfação geral dos turistas. Os resultados obtidos com a realização da presente investigação demonstram que, apesar dos critérios conforto e comodidades estarem - juntamente com o custo-benefício - entre os piores critérios avaliados pelos turistas, eles exercem grande influência na avaliação da qualidade dos serviços prestados pela hotelaria instalada em Florianópolis (SC).

Embora os critérios localização, funcionários e limpeza estejam entre os mais bem avaliados, os dois últimos também merecem atenção especial, uma vez que ainda não atingiram um nível de excelência na qualidade. O critério localização é o único que não se tem como melhorar, uma vez que não depende da gestão do hotel. Já o critério custo-benefício, acredita-se que, melhorando a qualidade dos demais critérios, a sua avaliação também será melhorada, uma vez que este critério é considerado, entre todos os demais, o mais subjetivo, o qual depende do juízo de valor inerente a cada turista.

\section{CONSIDERAÇÕES FINAIS}

A presente investigação buscou contribuir de forma teórica e empírica com as discussões relacionadas à qualidade dos serviços prestados por organizações hoteleiras, temática que vem sendo objeto de estudo de diversas pesquisas atualmente. Através da coleta de informações postadas no Booking. com, relacionadas aos hotéis localizados em Florianópolis (SC), foi possível identificar o grau de influência que os critérios de avalição dos turistas exercem na qualidade dos serviços prestados pela hotelaria local.

Os procedimentos metodológicos adotados conseguiram fazer com que o objetivo proposto fosse atingido. A quantidade de hotéis pesquisados, ou seja, todos os hotéis avaliados no Booking.com, traz robustez à pesquisa, podendo fornecer uma ideia geral do que os turistas que visitam Florianópolis (SC) pensam 
a respeito da qualidade dos serviços hoteleiros. As ferramentas estatísticas foram utilizadas para analisar os dados, encontrar os resultados, bem como confirmar os mesmos, a fim de demonstrar transparência na apresentação e na discussão das informações.

Após análise das informações, pode-se perceber que a hotelaria instalada em Florianópolis (SC) é composta, na sua maioria, por hotéis de 03 e 04 estrelas e que este tipo de classificação, baseada na tipologia por estrelas, apresentou-se como um meio adequado para categorizar os hotéis deste destino. Outra importante constatação é que todos os critérios avaliados, com exceção do Wi-Fi gratuito, exercem influência direta ou indireta na satisfação geral dos turistas.

Os dois critérios mais bem avaliados estão relacionados à localização e aos funcionários, corroborando com as pesquisas anteriores de autores como O'Connor (2010), Johann e Anastassova (2014) e Petry, Pickler e Tomelin (2016) no que se refere à localização; e Jeong e Jeon (2008), Stringam, Gerdes Jr. e Vanleeuwen (2010), Lima-Filho, Marchiotti e Quevedo-Silva (2012), Johann e Anastassova (2014) no que diz respeito aos funcionários. Entretanto, esses dois critérios não exercem influência direta sobre a avaliação dos turistas com relação aos demais critérios e, por isso, influenciando indiretamente na avaliação da satisfação geral dos mesmos.

Os critérios relacionados à comodidade e ao conforto, embora tenham obtido notas um pouco mais baixas, exercem grande influência na avaliação da qualidade dos serviços prestados pelos hotéis de Florianópolis (SC), uma vez que são considerados os maiores formadores da satisfação geral. Estes resultados corroboram com as pesquisas de Barcala, Díaz e Rodriguez (2009), Stringam, Gerdes Jr. e Valeeuwen (2010), Levy, Duan e Boo (2013), Li, Ye e Law (2013) e Jiang, Gretzel e Law (2014) no que se refere às comodidades; e com os estudos de O'Connor (2010), Stringam e Gerdes Jr. (2010), Stringam, Gerdes Jr. e Vanleeuwen (2010), Chaves, Gomes e Pedron (2013), Liu et al. (2013), Jiang, Gretzel e Law (2014) no que diz respeito ao conforto.

Sugere-se, para a realização de pesquisas futuras, que esta mesma metodologia de pesquisa seja aplicada nos hotéis deste mesmo destino, porém em diferentes épocas do ano, a fim de realizar um estudo longitudinal e, com isso, 
obter um maior número de informações a respeito da avaliação da satisfação dos turistas que frequentam o destino Florianópolis (SC). Sugere-se também que esta mesma metodologia seja aplicada em hotéis de outros destinos, nacionais e internacionais, a fim de realizar estudos comparativos e identificar de uma forma global a situação da qualidade dos serviços desempenhados pelas organizações hoteleiras cadastradas no Booking.com.

\section{REFERÊNCIAS}

Alonso-Almeida, M. M.; Rodríguez-Antón, J. M. \& Rubio-Andrada, L. (2012). Reasons for implementing certified quality systems and impact on performance: an analysis of the hotel industry. Service Industries Journal, 32(06), 919-936.

Ban, O. I. \& Mester, I. T. (2014). Using Kano two dimensional service quality classification and characteristic analysis from the perspective of hotels' clients of Oradea. Journal of Tourism, 18, 30-36.

Barcala, M. F.; Díaz, M. G.; Rodriguez, J. P. (2009). Factors influencing guests' hotel quality appraisals. European Journal of Tourism Research, 02(01), 25-40.

Berry, L. L. \& Parasuraman, A. (1995). Serviços de marketing: competindo através da qualidade. São Paulo: Maltese.

Black, H. G. \& Kelley, S. W. (2009). A storytelling perspective on online customer reviews reporting service failure and recovery. Journal of Travel \& Tourism Marketing, 26, 169-179.

Blesic, I.; Ivkov-Dzigurski, A.; Stankov, U.; Stamenkovic, I. \& Bradic, M. (2011). Research of expected and perceived service quality in hotel management. Journal of Tourism, 11, 05-13.

Booking.com. Disponível em: http://www.booking.com/content/about.pt-br.html Acesso: Abril de 2016.

Borges, I. R.; Pereira, G. M.; Matos, C. A. \& Borchardt, M. (2015). Analysis of the relationship between the satisfaction of consumers and the prices offered on site booking.com. Tourism \& Management Studies, 11(02), 64-70.

Browning, V.; So, K. K. F. \& Sparks, B. (2013). The influence of online reviews on consumers' attributions of service quality and control for service standards in hotels. Journal of Travel \& Tourism Marketing, 30, 23-40.

Chaves, M. S.; Gomes, R. \& Pedron, C. (2013). Analysing reviews in the web 2.0: Small and medium hotel in Portugal. Tourism Management, 33, 1286-1287. 
Chen, L-C. \& Tseng, C-Y. (2012). Benefits of Cross-Functional Training: Three Departments of Hotel Line Supervisors in Taiwan. Journal of Hospitality and Tourism Management, 19, 01-08.

Cronin, J. J. \& Taylor, S. A. (1992). Measuring service quality: a reexamination and extension. Journal of Marketing, 56(03), 55-68.

Curakovic, D.; Sikora, I.; Garaca, V.; Curcic, N. \& Vukosav, S. (2013). The degree of consumer satisfaction with hotel services. Journal of Tourism, 15, 06-11.

Falces, D. C.; Sierra, D. B.; Becerra, G. A. L. \& Briñol, T. P. (1999). HOTELQUAL: una escala para medir calidad percibida en servicios de alojamiento. Estudios Turísticos, (139), 95-110.

Gémar, G. \& Quintero, J. A. J. (2015). Text mining social media for competitive analysis. Tourism \& Management Studies, 11(01), 84-90.

Gidumal, J. B. \& González, S. M. \& Valcarcel, B. G. L. (2013). A social media analysis of the contribution of destination to client satisfaction with hotels. International Journal of Hospitality Management, 35, 44-47.

González, S. M.; Gidumal, J. B. \& Valcarcel, B. G. L. (2013). Online customer reviews of hotels: As participation increases, better evaluation is obtained. Cornell Hospitality Quarterly, 54(03), 274-283.

Grobelna, A. \& Marciszewska, B. (2013). Measurement of service quality in the hotel sector: the case of Northern Poland. Journal of Hospitality Marketing \& Management, 22(03), 313-332.

Hair Jr, J. F.; Anderson, R. E.; Tatham, R. L. \& Black, W. C. (2009). Análise multivariada de dados. Porto Alegre: Bookman.

Instituto Brasileiro de Geografia e Estatística (IBGE). Disponível em: http://cod.ibge.gov. br/4J6. Acesso: Maio de 2016.

Jeong, M. \& Jeon, M. M. (2008). Customer reviews of hotel experiences through consumergenerated media (CGM). Journal of Hospitality \& Leisure Marketing, 17(01-02), 121-138.

Jiang, J.; Gretzel, U. \& Law, R. (2014). Influence of star rating and ownership structure on brand image of mainland china hotels. Journal of China Tourism Research, 10(01), 69-94.

Johann, M. \& Anastassova, L. (2014). The perception of tourism product quality and tourist satisfaction: the case of polish tourists visiting Bulgaria. European Journal of Tourism Research, 08, 99-114.

Knutson, B.; Stevens, P.; Wullaert, C.; Patton, M. \& Yokoyama, F. (1990). LODGSERV: a service quality index for the lodging industry. Hospitality Research Journal, 14(02), 227-284. 
Kuo, N-T.; Chang, K-C.; Cheng, Y-S. \& Lai, C-H. (2013) Investigating the effect of service quality on customer loyalty in the hotel industry: the mediating role of customer satisfaction and the moderating roles of service recovery and perceived value. Journal of China Tourism Research, 09(03), 257-276.

Kuo, N-T.; Chang, K-C.; Chen, M-C. \& Hsu, C-L. (2012). Investigating the effect of service quality on customer post-purchasing behaviors in the hotel sector: the moderating role of service convenience. Journal of Quality Assurance in Tourism \& Hospitality, 13, 212-234.

Law, R. \& Yip, R. (2010). A study of satisfaction level of Hong Kong tourists with Hot Springs Hotels and Resorts in Guangdong, China. FIU Hospitality Review, 28(01), 83-107.

Lee, C. C. \& Hu, C. (2008). Analyzing hotel customers' e-complaints from an internet complaint forum. Journal of Travel \& Tourism Marketing, 17(02-03), 167-181.

Levy, S. E.; Duan, W. \& Boo, S. (2013). An analysis of one-star online reviews and responses in the Washington, D.C., lodging market. Cornell Hospitality Quarterly, 54(01), 49-63.

Li, D. (2014). A Study on the impact of other customers' behavior in hotel on customer perceived service quality. Tourism Tribune, 29(04), 48-54.

Li, H.; Ye, Q. \& Law, R. (2013). Determinants of customer satisfaction in the hotel industry: an application of online review analysis. Asia Pacific Journal of Tourism Research, 18(07), 784-802.

Lima-Filho, D. O.; Marchiotti, I. Z. \& Quevedo-Silva, F. (2012). Expectativas versus satisfação dos consumidores da rede hoteleira de Campo Grande - MS. Turismo em Análise, 23(01), 54-77.

Limberger, P. F.; Anjos, F. A.; Meira, J. V. S. \& Anjos, S. J. G. (2014). Satisfaction in hospitality on TripAdvisor: an analysis of the correlation between evaluation criteria and overall satisfaction. Tourism \& Management Studies, 10(01).

Linnes, C.; Kowalski, P.; Lema, J.; Lam, W. \& Agrusa, J. (2014). Social media and technology: the influence on Hawaii's hotel. Consortium Journal of Hospitality and Tourism, 19(02), 54-73.

Liu, S.; Law, R.; Rong, J.; Li, G. \& Hall, J. (2013). Analyzing changes in hotel customers' expectations by trip mode. International Journal of Hospitality Management, 34, 359-371.

Marinescu, R. C. \& Ispas, R. (2012). Achieving sustainable tourism through customer satisfaction. In: University of Oradea Economic Science Series, 2012, Oradea. Anais... Oradea, Romania.

Markovic, S. \& Jankovic, S. R. (2013). Exploring the relationship between service quality and customer satisfaction in Croatian hotel industry. Tourism and Hospitality Management, 19(02), 149-164. 
Martins, G. A. \& Theóphilo, C. R. (2009). Metodologia da investigação científica para ciências sociais aplicadas. 2. ed. São Paulo: Atlas.

Mei, A. W. O.; Dean, A. M. \& White, C. J. (1999). Analyzing service quality in the hospitality industry. Managing Service Quality, 09(02), 136-143.

Noone, B. F. \& McGuire, K. A. (2013). Effects of price and user-generated content on consumers' repurchase evaluations of variably priced services. Journal of Hospitality \& Tourism Research. Doi: 10.1177/1096348012461551.

O'Connor, P. (2010). Managing a hotel's image on TripAdvisor. Journal of Hospitality Marketing \& Management, 19, 754-772.

Ong, B. S. (2012). The perceived influence of user reviews in the hospitality industry. Journal of Hospitality Marketing \& Management, 21, 463-485.

Petry, T. R. E.; Pickler, C. M. \& Tomelin, C. A. (2016). A percepção dos hóspedes de negócio quanto ao desempenho da qualidade dos serviços prestados nos hotéis de Florianópolis: uma análise a partir do conteúdo gerado no website booking.com. Turismo Visão e Ação, 18(02), 327-352.

Portal da Ilha. Disponível em: http://www.portaldailha.com.br Acesso: Maio de 2016.

Prefeitura Municipal de Florianópolis (PMF). Disponível em: http://www.pmf.sc.gov.br. Acesso: Maio de 2016.

Silva, A. J. H. \& Teixeira, R. M. (2007). Ambiente Competitivo e Vantagem Competitiva a partir de uma Abordagem Integradora: Estudo de Caso no Setor Hoteleiro em Curitiba. Turismo Visão e Ação, 09(01), 19-36.

Souza, E. C.; Meira, J. V. S. \& Maske, D. C. (2012). A medição da qualidade dos serviços prestados em hotéis de Balneário Camboriú, SC: uma aplicação do modelo SERVQUAL. Revista Rosa dos Ventos, 04(04), 544-555.

Stringam, B. B. \& Gerdes Jr, J. (2010). An analysis of word-of-mouth ratings and guest comments of online hotel distribution site. Journal of Hospitality Marketing \& Management, 19, 773-796.

Stringam, B. B.; Gerdes Jr, J. \& Vanleeuwen, D. D. (2010). Assessing the importance and relationships of ratings on user-generated travel reviews. Journal of Quality Assurance in Hospitality \& Tourism, 11, 73-92.

Stupariu, M. I. \& Josan, I. (2014). The quality of hotel services. Case study: the county seat municipalities of the North-West Development Region. GeoJournal of Tourism and Geosites, 14(02), 207-214. 
TripAdvisor. Disponível em: http://www.tripadvisor.com.br. Acesso: Maio de 2016.

Wilkins, H.; Merrilees, B. \& Herington, C. (2010). The determinants of loyalty in hotels. Journal of Hospitality Marketing \& Management, 19, 01-21.

Xiang, Z. \& Gretzel, U. (2010). Role of social media in online travel information search. Tourism Management, 31, 179-188.

Ye, Q.; Law, R. \& Gu, B. (2009). The impact of online user reviews on hotel room sales. International Journal of Hospitality Management, 28(01), 180-182.

Zehrer, A.; Crotts, J. C. \& Magnini, V. P. (2011). The perceived usefulness of blog postings: An extension of the expectancy-disconfirmation paradigm. Tourism Management, 32, 106-113.

Zhen, L. \& Zhu, F. (2010). Perceptions of Chinese and international tourists on China hotel service quality. Journal of China Tourism Research, 06, 73-82.

Zheng, T.; Youn, H. \& Kincaid, C. S. (2009). An analysis of customers' e-complaints for luxury resort properties. Journal of Hospitality Marketing \& Management, 18, 718-729.

Zhong, J.; Chen, X. \& Xie, L. (2010). China hotel quality competitiveness assessment: a quality competition index approach. Journal of China Tourism Research, 06(02), 145-163. 